\title{
PROBABILISTIC TSUNAMI HAZARD ASSESSMENT (PTHA) AND PTHA-BASED TSUNAMI SCENARIO AT A PACIFIC COAST OF TOHOKU
}

\author{
Naoto Kihara, Central Research Institute of Electric Power Industry, kihara@criepi.denken.or.jp \\ Hideki Kaida, Central Research Institute of Electric Power Industry, h-kaida@criepi.denken.or.jp \\ Tatsuto Kimura, Tokyo Electric Power Service Co., Ltd., kimura tatsu@tepsco.co.jp \\ Naoki Fujii, Tokyo Electric Power Service Co., Ltd., dfujii@tepsco.co.jp \\ Keiichi lizuka, Tokyo Electric Power Service Co., Ltd., iizuka@tepsco.co.jp
}

\section{INTRODUCTION}

When planning tsunami disaster mitigation and designing important infrastructure from the viewpoint of tsunami resistance in coastal areas, the scale and frequency of tsunamis that will arrive at coastal areas in future are important information. On the other hand, there are large uncertainties in predicting future tsunamis, and thus it is difficult to predict a future tsunami correctly. The technology of probabilistic tsunami hazard assessment (PTHA) has been proposed to evaluate the relationship between the height and frequency of tsunamis that will arrive at coastal areas in future. To consider the uncertainties of the prediction in the PTHA, the logic-tree approach is often adopted. In this approach, both epistemic and aleatory uncertainties are considered systematically. The epistemic uncertainties are caused by lack of knowledge and the aleatory uncertainties are variabilities due to natural randomness. In the logic-tree approach, the epistemic uncertainties are expressed by tree branches and the aleatory uncertainties are expressed by the probabilistic density functions of predicted tsunami heights. By carrying out PTHA, we can obtain a hazard curve, which expresses the relationship between the tsunami height and annual frequency of exceedance. Recently, methodologies by which PTHA-based-tsunami-scenarios are determined have been proposed. By using tsunami scenarios, detailed inundation processes and patterns can be evaluated. In this study, we apply the technologies of PTHA to the pacific coast of Tohoku, Japan. Then, we determine PTHA-based tsunami scenarios, that overflow a seawall constructed at the target coast and can be used for the evaluation of inundation processes.

\section{PROBABILISTIC TSUNAMI HAZARD ASSESSMENT} AT A PACIFIC COAST OF JAPAN

The evaluated target coast is located in the northeastern coastal region of Japan. At the coast, seawalls with T.P.+9.7 m are now being constructed following the disasters caused by the 2011 Tohoku earthquake tsunami. The methodology of PTHA adopted in this study is the logic -tree approach and is based on that proposed by JSCE (2011). The earthquake-occurrence areas considered in this study are shown in Figure 1, and logic trees are set for each earthquake-occurrence area. In the logic trees, branches representing epistemic uncertainties for the location of the tsunami source, the magnitude, the fault model, the occurrence interval, and the stress drop for the occurrence of an earthquake are set. In accordance with the logic trees, tsunami simulations are carried out, and the number of simulation cases is 165 in this study. The evaluated hazard curves for the tsunami heights in front of the seawall are shown in Figure 2. The hazard curve shows that the annual frequency of exceedance of tsunami-occurrence, which overflow the seawall, is
$8 \times 10^{-4}$

\section{DETERMINATION OF TSUNAMI SCENARIOS}

For the identification of a dominant tsunami that will arrive at the target coast in a target range of tsunami height. In this study, the target range of tsunami height is defined as the range from the top level of the seawall to T.P.+25 m. The hazard curve in front of the seawall shows that the dominant earthquake-occurrence areas in this range are JTT and JTC2 (Figure 2). Furthermore, by investigating relative contributions of each magnitude and source to the hazard and the similarity of the wave forms, tsunami scenarios are determined.

\section{REFERENCES}

Japan Society of Civil Engineering (2011): A method for probabilistic tsunami hazard assessment, http://committees.jsce.or.jp/ceofnp/system/files/PTHA20 111209 0.pdf (accessed on 25 December, 2015, in Japanese).

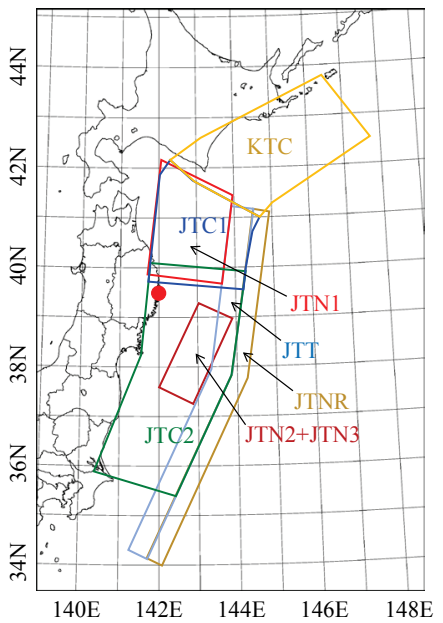

Figure 1 - Earthquake-occurrence areas considered in the PTHA of this study. Solid circle is the target coast.

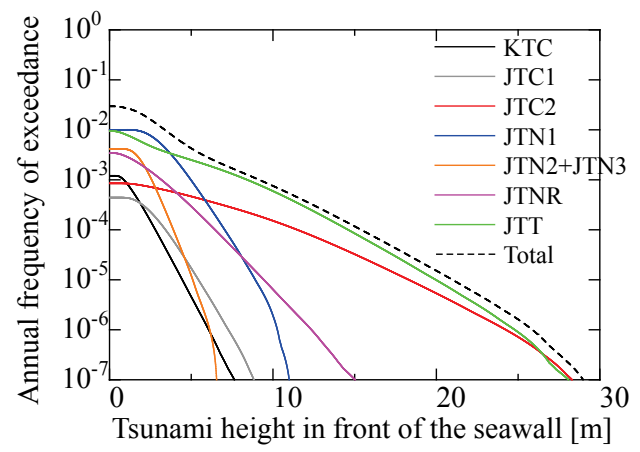

Figure 2 - Hazard curve in front of the seawall 